\title{
Monitoring robotic swarm systems under noisy conditions using an effective fault detection strategy
}

\author{
Fouzi Harrou, Member, IEEE, Belkacem Khaldi, Ying Sun, and Foudil Cherif
}

\begin{abstract}
Fault detection in robotic swarm systems is imperative to guarantee their reliability, safety, and to maximize operating efficiency and avoid expensive maintenance. However, data from these systems are generally contaminated with noise, which masks important features in the data and degrades the fault detection capability. This paper introduces an effective fault detection approach against noise and uncertainties in data, which integrates the multiresolution representation of data using wavelets with the sensitivity to small changes of an exponentially weighted moving average scheme. Specifically, to monitor swarm robotics systems performing a virtual viscoelastic control model for circle formation task, the proposed scheme has been applied to the uncorrelated residuals form principal component analysis model. A simulated data from ARGoS simulator is used to evaluate the effectiveness of the proposed method. Also, we compared the performance of the proposed approach to that of the conventional principal component-based approach and found improved sensitivity to faults and robustness to noises. For all the fault types tested-abrupt faults, random walks, and complete stop faults-our approach resulted in a significant enhancement in fault detection compared with the conventional approach.
\end{abstract}

Keywords-Robust detection, robotic swarm systems, viscoelastic control model, Wavelet, multiresolution.

\section{INTRODUCTION}

$\mathbf{S}$ WARM intelligence has been remarkably observed in many biological organisms such as social insects and order living animals [1]. With swarm intelligence, complex swarming behaviours can emerge from simple interactions within individuals of a swarm in addition to their interaction with their environment [2]. Inspired by nature swarm intelligence (e.g., insect swarms and flocks of birds), a new line of research has been founded called "swarm robotics" [3]. In swarm robotics, a large group of relatively simple robots can perform specific tasks in a collaborative and cooperative manner by sharing interest in swarm intelligence features such as selforganization, decentralization, scalability, and robustness [3].

Despite the robustness and scalability that characterize swarm robotics systems, it has been shown that these systems can lead to undesirable results while accomplishing the desired task [4], [5]. This can mainly due to one or more faulty robots that commonly result in performance degradation of the entire swarm system. Practically, failures can be resulted from faults

F. Harrou and Y. Sun are with King Abdullah University of Science and Technology (KAUST) Computer, Electrical and Mathematical Sciences and Engineering (CEMSE) Division, Thuwal, 23955-6900, Saudi Arabia e-mail: fouzi.harrou@kaust.edu.sa

B. Khaldi and F. Cherif are with LESIA Laboratory, Department of Computer Science, University of Mohamed Khider, R.P. 07000 Biskra, Algeria in the robot components such as bugs in its software controller or damages in its sensors/actuation devices; it can also due to topological faults such as damaged communication links between certain robots of the swarm.

Recent studies have revealed that swarm robotics systems are less tolerant to partial failure robot(s) when compared to complete failure robot(s) [4]. As an example, a failure in a robot' component (i.e. motors) with the other sub-components functioning well, can effect the motion of the entire swarm. Bjerknes and Winfield [5] evaluated and analyzed the effect of such failure and other failures modes in a wireless connected robot swarms system both in simulation and real experimental platforms. The failures modes concerned in this analysis involved a robot' power failure mode, a robot' IR sensor failure mode, and a robot' motors failure mode. The reliability of the swarm robots was analyzed by understanding which failure mode could be the serious source affecting the performance of the overall swarm system. The study revealed that a potentially serious consequence of producing a partially failed robot that have an effect in the swarm movement is highly caused by robot(s) motor failures. Therefore, Bjerknes and Winfield [5] concluded that the consequence of partial robot failures should be critically considered when analyzing fault tolerance in robot swarms, and when designing robots swarm controllers.

Detecting such failures in such swarm systems become a challenging aspect for the engineers and specialists of the domain, and till now an increased attention for monitoring swarm robotics is leading to the development of many fault detection techniques, which are classified into endogenous and exogenous fault detection methods [6], [7]. In endogenous fault detection methods, a single robot makes its own decision if he is fault-free by detecting faults that may occur in its own components. A few works approaching such methodology have been reported; Skoundrianos et al. [8] diagnosed faults in a mobile robot' wheels using a local model neural network. Christensen et al. [9] synthesized task-dependent monitoring modules in s-bot robots using a time-delay neural network. Mokhtar et al. [10] adjusted a fault detection method inspired from the functioning of dendritic cells in the immune system. However, a misleading diagnosis might be resulted in these approaches due to ignoring the interaction between robots. For example, anomalies might not be detected by a robot itself, and even it is successfully detected it cannot be signalled to the other robots of the swarm if anomalies occur in the robot communication device.

On the other hand, in exogenous fault detection methods, the swarm collaboratively makes decision in detecting faults 
by taking into account the available information of the swarm neighborhood [7]. This means that faults occurred in a robot's components could be detected by other robots of the swarm. Christensen et al. [7], suggested a firefly-inspired exogenous monitoring method, which has revealed the ability of real robots to detect non-operational robots in the swarm. Tarapore et al. [11] implemented a swarm robotics exogenous fault detection approach based on Artificial Immune System (AIS) with testing its efficiency on various case studies that included dispersing, aggregating, harming, and flocking. In the same vision, Jakimovski et al. [12] introduced an AIS method, inspired by the T-Cell Receptor and intracellular signaling network procedures to identify faults in swarm systems. In another works, Millard et al. [6] developed an internal prediction modeling approach in every robot and compare it with the real performance of other robots in the swarm to detect faults in run-time.

To develop endogenous and exogenous monitoring methods, either an empirical implicit model or a mathematical model can be used. For detecting faults in mathematical model-based approaches, generally, the actual performances of the inspected swarm are compared with predicted performances obtained from an analytical model of the swarm [13]. Regrettably, designing such precise models to monitor robots swarm systems, can be hard and time-consuming. An alternative to these models is data-based models, which are appropriate in cases where an explicit model is absent, and the only available resource for anomaly detection is measurement signals [14]. In databased methods, relevant features in the design of monitoring procedures are efficiently extracted based on empirical models that are obtained from the available process measurements. In such methods, and depending on the quality of the available input data, a minimal prior knowledge about process physics is required. In fact, computational intelligence and machine learning approaches are mainly the basics of data- driven methods. To reach these objectives, multivariate statistical process control schemes are among the techniques that have been widely used recently [15].

It is essential to be capable to timely detect potential faults in the inspected robot swarm to maximize operating profitability and avoid costly maintenance. Yet little work exists on multivariate statistical monitoring for robotic swarm systems. Recently, in [15], a statistical procedure for monitoring robot swarms has been developed and was successfully applied in a simulated version of foot-bot robots [16]. This approach combines the benefits of a principal component analysis (PCA) modeling and the ability of an exponentially weighted moving average scheme (EWMA) scheme to uncover faults in swarm robots. PCA is used to compute residuals, and then employ EWMA approach to uncover any fault. However, EWMAbased charts don't consider the multiscale nature of data. However, the noise present in measurements can mask important features in data or lead to misleading indications by increasing false alarms and missed detections. In other words, the quality of the detection approach degrades with noisy data because faults and important features can easily get masked by the noise. The measurement noise is spread in time-frequency domain which needs effective feature extraction from time as well as frequency scale. Multiresolution representation of data via wavelets is an efficient feature extraction tool that has the ability to feature/noise separation and decorrelating time series data [17]. Accounting for noise and uncertainty in the data should be considered when designing fault detection schemes. The objective is to exploit the advantages of waveletbased multi-scale representation and an EWMA scheme to address the problem of measurement noise in data and to better detect defects in robot swarms. A framework merging the advantages of EWMA monitoring scheme and the wavelet decomposition with multiscale representation is proposed and used in the context of robust fault detection in robot swarms. This procedure will be called the WM-EWMA chart hereafter.

The virtual viscoelastic control (VVC) modeling procedure implemented for the circle formation of a robot swarm is briefed in the following section. Then the PCA technique is introduced in Section III. In Section IV, multiscale representation of data with wavelet and its use in fault detection with EWMA, and the proposed monitoring approach are described. In Section $\mathrm{V}$ we evaluate the efficiency of the developed technique using measurements obtained from the ARGoS simulator, and conclusions are given in Section VI.

\section{VIRTUAL VISCOELASTIC CONTROL MODELING APPROACH}

In the VVC model, a virtual physics approach is followed to self-organize a swarm robotics system into a circle formation [18]. The model has been also effectively used as a proximal controller to aggregate robots swarm into self-organized patterns [19]. The idea behind the VVC model is to model the interactions between the robots, and hence the movement of the overall swarm, using virtual viscoelastic forces. A VVC configuration of a circle of radius $r$, formed by four foot-bot robots, is illustrated in Fig. 1.

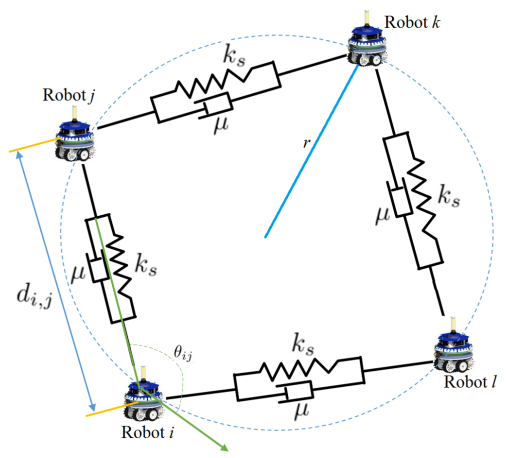

Fig. 1: A VVC configuration of a circle formed by 4-robots.

A foot-bot (see Figure 2) is considered as a differential wheeled robot of approximately $14.7 \mathrm{~cm}$ high and $8.5 \mathrm{~cm}$ wide [20]. It can move with a maximum forward speed of $13 \mathrm{~cm} / \mathrm{s}$. The robot is well equipped with a set of actuators and sensors that can be used to synthesize controllers for many swarming behaviors [21]. With the VVC model, we specically 
used the RAB (range and bearing) communication device for inter-robots communications and two-wheeled actuators to independently control the forward speed of the robots right and left wheels.

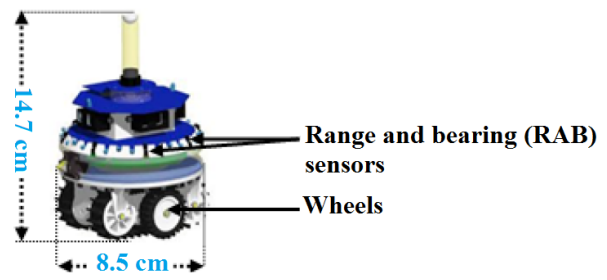

Fig. 2: The foot-bot robot, a CAD model.

The total virtual viscoelastic force $F_{i}^{v v c}$ exerted on a robot $i$ is obtained using Equation (1). Then basing on that force, the desired forward speeds $v_{r i}$ and $v_{l i}$ of the right and the left wheels of the robot is given in Equation (2).

$$
\begin{gathered}
F_{i}^{v v c}=\sum_{j=1}^{n}\left(k_{s}\left(d_{i, j}-d_{0}\right) \hat{d}_{i j}+\mu\left(v_{i}-v_{j}\right),\right. \\
{\left[\begin{array}{c}
v_{l_{i}} \\
v_{r_{i}}
\end{array}\right]=\left[\begin{array}{cc}
1 & \frac{b}{2} \\
1 & \frac{-b}{2}
\end{array}\right]\left[\begin{array}{c}
v_{i} \\
\omega_{i}
\end{array}\right],}
\end{gathered}
$$

with

$$
\omega_{i}=k_{\omega} \angle F_{i}^{v v c}, v_{i}=\frac{v_{\max }}{\sqrt{\left|\omega_{i}\right|+1}}
$$

Where in equation (1), $d_{0}=2 r \sin (\pi /(n+1))$ is the equilibrium length of the spring, $n$ is the number of neighbors, $k_{s}$ is the spring constant, $d_{i, j}$ is the current length of the spring between two interacting robots, $\hat{d}_{i j}$ is the unit vector indicating the direction of the virtual viscoelastic force, and $\mu$ is the damping coefficient. $v_{i}$ and $v_{j}$ are the velocities of the interacting robots $i$ and $j$.

Whereas in equation (2), $b$ is the distance between the robot' wheels, $\omega_{i}$ is the robot' angular velocity, and $v_{i}$ is the robot' forward speed.

Finally in equation (3), $k_{\omega}$ is a gain constant, $\angle F_{i}^{v v c}$ refers to the angle formed by the force $F_{i}^{v v c}$, and $v_{\max }$ is the maximum allowed forward speed.

In a foot-bot robot, the VVC model is achieved using the robot' $\mathrm{RAB}$ device (range and bearing sensing and communication device). With this device and within a maximum range $D_{r}$, messages can be sent and received to (from) neighboring robots. The constantes used for the implementation of the VVC model in the foot-bot robot is outlined in Table I

The pseudo code for implementing the VVC model on each foot-bot robot is implemented in the the ARGoS simulator [22], a multi- physics customizable robot simulator that can simulate large-scale swarms of robots. Next a description of each pseudo code line in the VVC algorithm is given.

1) First, the different constants related to the VVC model and which are pointed in Table I are initialized. Also the robot forward speed $v_{i}$ is initialized to 0 .
TABLE I: Values of parameters used in VCC model.

\begin{tabular}{l|l|l}
\hline Parameter & Description & Value \\
\hline$b$ & Inter-wheels distance & $0.14 \mathrm{~m}$ \\
\hline$v_{\max }$ & Maximum forward speed & $0.10 \mathrm{~m} / \mathrm{s}$ \\
\hline$k_{s}$ & Spring gain constant & 1.9 force unit \\
\hline$\mu$ & Damper gain constant & 1.25 force unit \\
\hline$k_{\omega}$ & Angular speed gain & $1.5 \% / \mathrm{s}$ \\
\hline$r$ & Radius of the desired circle & $1.8 \mathrm{~m}$ \\
\hline
\end{tabular}

2) Next, at each ARGoS time step, $t s$, which is commonly set to $0.1 s$, ARGoS updates the robot's state by executing the control steps from line 3 to 14 .

3) Using the robot RAB device, the current forward speed $v_{i}$ of the robot is sent to the neighbors.

4) When sending a data to neighbors via the RAB device, the robot immediately can get the number $n$ of the sensed neighbors.

5) Compute the equilibrium length of the spring $d_{0}$ which represents the side length of the circumcircle to be formed [18].

6) Initialize the force $F_{i}^{v v c}$ to $\overrightarrow{0} . F_{i}^{v v c}$ is a vector of $x$ and $y$ components.

7) Using again the RAB device, the robot iterates over the received data that are sent by neighbors and executes the control steps between line 8 and 10 .

8) Get the velocity $v_{j}$ sent by neighbor $j$ using the RAB device.

9) With the RAB device, information such as the range $\left(d_{i j}\right)$ and the bearing $\left(\theta_{i j}\right)$ from which the data is received can be measured.

10) Compute the virtual viscoelastic force exerted by the neighbor $j$ with updating the total virtual force vector $F_{i}^{v v c}$ using eq. 1 .

11) End of iterating over each data in the RAB.

12) Upon computing the total virtual viscoelastic force vector $F_{i}^{v v c}$, the force is used to calculate both the forward speed $v_{i}$ and the angular speed $\omega_{i}$ of the focal robot using eq. 3 .

13) Then using eq.3, the robot computes the forward speed of its left and right wheels.

14) Finally, the robot actuates the speed of its left and right wheels by the velocities calculated in step control 13 .

15) End iterating over each time step.

\section{FEATURE EXTRACTION VIA PCA}

PCA has become a well-reputed modeling procedure used for extracting relevant information from multivariate data [14], [23], [24]. PCA projects the process data at each time point into lower dimensional space revealing inherent data structure [25]. Let $\mathbf{X}=\left[\mathbf{x}_{1}^{T}, \ldots, \mathbf{x}_{n}^{T}\right]^{T} \in R^{n \times m}$ be a scaled measurement matrix with $n$ measurements and $m$ variables. The data matrix $\mathbf{X}$ is factorized using PCA scheme into two orthogonal parts,

$$
\mathbf{X}=\mathbf{T} \mathbf{W}^{T}=\sum_{i=1}^{k} t_{i} w_{i}^{T}+\sum_{i=k+1}^{m} t_{i} w_{i}^{T}=\widehat{\mathbf{X}}+\mathbf{E}
$$


where $\widehat{\mathbf{X}}$ is the approximated data and $\mathbf{E}$ is a residual data. $\mathbf{T}=\left[t_{1} t_{2} \cdots t_{m}\right] \in R^{n \times m}$ and $\mathbf{W} \in R^{m \times m}$ represent a matrix of the transformed uncorrelated variables (principal components) and loading matrix, respectively. Given certain correlation (redundancy) in data $\mathbf{X}$, the first ' $k$ ' PCs (where $k<m$ ) are abale to capture most of the variability in $\mathbf{X}$.

The loading matrix is usually computed via Singular Value Decomposition (SVD) of the covariance matrix $\mathbf{S}$ of the input data $\mathbf{X}$ as:

$$
\mathbf{S}=\frac{1}{n-1} \mathbf{X}^{T} \mathbf{X}=W \Lambda W^{T} \text { with } W W^{T}=W^{T} W=I_{n} .
$$

Here, $\Lambda=\operatorname{diag}\left(\sigma_{1}^{2}, \ldots, \sigma_{m}^{2}\right)$ is a diagonal matrix comprising the decreasingly ordered eigenvalues of $\mathbf{S}$. The eigenvalues $\lambda_{i}$ are equal to the variance of the PC $t_{i}, \sigma_{i}^{2}$. From equation (4)

$$
\mathbf{X}=\widehat{\mathbf{T}} \widehat{\mathbf{W}}^{T}+\widetilde{\mathbf{T}} \widetilde{\mathbf{W}}^{T}=\widehat{\mathbf{X}}+\mathbf{E}
$$

where $\widehat{W} \in R^{m \times k}$ comprised the eigenvectors corresponding to the first $k$ largest eigenvalues in $\Lambda \in R^{k \times k}$, and $\widetilde{W} \in R^{m \times(m-k)}$ represents the remaining $m-k$ eigenvectors associated to the rest of eigenvalues. $\widehat{\mathbf{T}}=\left[t_{1}, \ldots, t_{k}\right]$ is the principal component score matrix $(n \times k)$, which describes the values of variables in the transformed $n \times k$ basis space spanned by $\widehat{\mathbf{W}}$. Here $\widetilde{\mathbf{T}}=$ $\left[t_{k+1}, \ldots, t_{m}\right]$ is obtained by choosing the last $m-k$ PCs in $\mathbf{T}$ such that $\widetilde{\mathbf{T}}$ represents only the variability of random errors.

Here, we use CPV procedure to select the number of retained PCs, $C P V(l)=\frac{\sum_{i=1}^{k} \lambda_{i}}{\sum_{i=1}^{m} \lambda_{i}} \times 100$. In this CPV procedure, the number of PCs is determined by counting PCs until the cumulative variance explains the desired percentage (e.g., 90\%) of the total variance. In [15], a data-driven approach based on PCA model for monitoring a robot swarm has been developed. In this approach, a PCA model was employed for generating residuals and an EWMA chart was used to detect faults in the monitored robot swarm (Figure 3). However, the noise present in measurements can mask important features in data or lead to misleading indications by increasing false alarms and missed detections. In addition, EWMA-based charts don't consider the multiscale nature of data. Robustness of a monitoring system is crucial to operating in noisy environments whilst preserving sensitivity to anomalies. The measurement noise is spread in time-frequency domain which needs suitable feature extraction from both time and frequency scale. To cope with the aforementioned limitations, we will propose an approach based on the fusion of the EWMA scheme with wavelet-based multiscale analysis for further enhanced detection results.

\section{ENHANCED MONITORING USING THE MULTISCALE EWMA ALGORITHM}

This section presents the proposed approach for fault detection in a robot swarm. This technique mixes the EWMA technique with wavelet-based multiscale representation.

A. EWMA chart

EWMA scheme is an important univariate quality control procedure, which has been used in several disciplines to online monitor the mean of production processes [26]-[28]. An

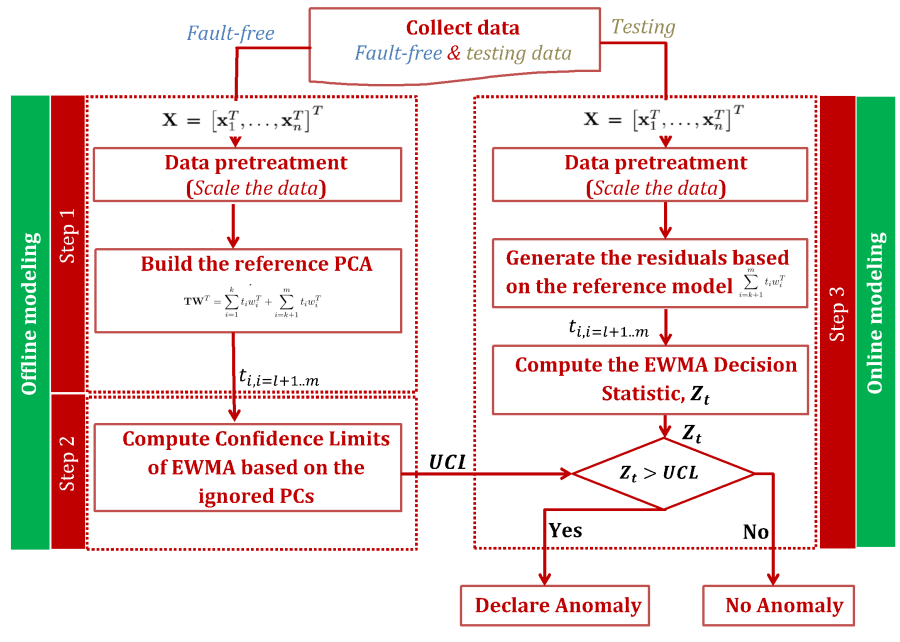

Fig. 3: PCA-EWMA monitoring procedure.

advantage of EWMA scheme is aggregating all available information from past and actual samples in the decision rule. The EWMA fault decision statistic is computed as follows [26]:

$$
z_{t}=v x_{t}+(1-v) z_{t-1}
$$

where $x_{t}$ is the measurement at time instant $t$, the initial value, $z_{0}$ is the fault-free mean of data, $\mu_{0}$, and $v(0<v \leq 1)$ is a smoothing parameter. For this chart, the general guidelines for selecting $v$ is: small $v$ values are appropriate for detecting small faults and large $v$ values are appropriate for detecting large faults. The upper control limit $U$, and the lower control limit Lof the EWMA scheme can then be defined by

$$
U, L=\mu_{0} \pm \kappa \sigma_{0} \sqrt{\frac{v}{(2-v)}\left[1-(1-v)^{2 t}\right]},
$$

where $\sigma_{0}$ is the standard deviation of the fault-free data, and $\kappa$ is the width of the control limits. When $z_{t}$ is within the decision thresholds, then the swarm is considered normal. Otherwise, a signal of a fault is given.

\section{B. Wavelet-based multiscale representation}

Data from swarm robotics systems are generally tainted with noise. The presence of a large amount of noise in the measurements can mask the important features and anomalies in the data by erroneously providing the wrong reading from sensors. Accordingly, the anomaly detection capability of a monitoring system can be degraded. To cope with these problems, wavelet-based multiscale modeling of data plays a key role in extracting useful feature and reducing noise effect from the contaminated data [29].

Generally speaking, by using the Discrete Wavelet Transform (DWT) a signal can be expressed as a combination of approximation and detail coefficients [30]. Towards this end, a set of scaling functions and a set of wavelet functions, which are respectively linked to a low pass filter $H$ and high pass filter $G$, are utilized for approximating the signal at various fine scales. Approximation coefficients result from a linear 
convolution between the signal and the low pass filter and after performing downsampling by 2 . In other words, approximation coefficients are obtained from the projection of the original time series a set of orthonormal scaling functions defined as,

$$
\phi_{j, k}(t)=\sqrt{2^{-j}} \phi\left(2^{-j} t-k\right), j=1, \ldots, J, k \in \mathbb{Z} .
$$

Then, approximation signal, $D_{j}(t)$ at level $j$ has the following expression,

$$
A_{j}(t)=\sum_{k=1}^{n 2^{-j}} a_{j k} \phi_{j k}(t),
$$

where $a_{j k}$ is the wavelet coefficient, $n$ is the number of observations in the original signal, and $j$ is the number of scale [31]. Similarly, detail coefficients are obtained by performing downsampling of 2 of a linear convolution between signal and a high pass filter. In other words, detail coefficients are obtained by the projection of the original time series a set of wavelet basis functions defined as,

$$
\psi_{j, k}(t)=\sqrt{2^{-j}} \psi\left(2^{-j} t-k\right), j=1, \ldots, J, k \in \mathbb{Z},
$$

where the coarsest scale $J$ usually termed the decomposition level. Therefore, the detail coefficient at level $j$ can be written as

$$
D_{j}(t)=\sum_{k=1}^{n 2^{-j}} d_{j k} \psi_{j k}(t),
$$

where $d_{j k}$ are the scaling coefficients. A signal can be expressed as [30]:

$$
x(t)=A_{J}(t)+\sum_{j=1}^{J} D_{j}(t) .
$$

The decomposition procedure of a signal based on DWT is presented in Figure 4.

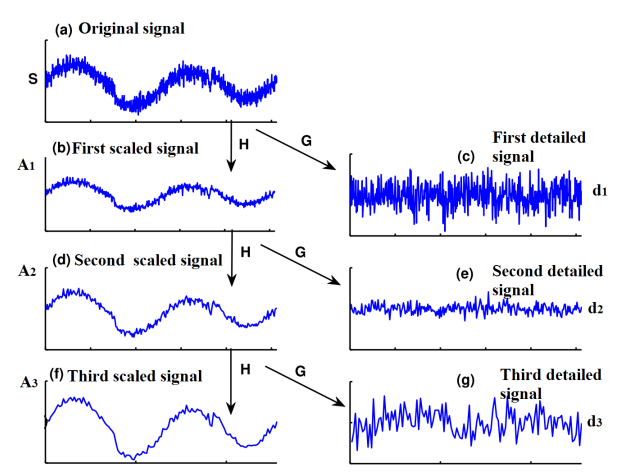

Fig. 4: Illustration of wavelet decomposition and separation of stochastic and deterministic components.

Figure 4 shows a three-level DWT of the signal $S, A_{1}$ and $d_{1}$ are respectively approximation and detail coefficients at level $1, A_{1}$ is further represented by the summation of approximation and detail coefficients at level $2, A_{2}$ and $d_{3}$, respectively; and so on.
Of course, wavelet-based filtering is a powerful procedure for effectively separating noise-feature in the data [32]. Here, we exploit this advantage to develop a superior multiscale univariate anomaly detection method and show how the method can be applied successfully to fault detection.

\section{Advantages of multiresolution representation}

This subsection is dedicated to briefly presenting the main advantages of wavelet-based multiscale representation.

1) Decorrelation of autocorrelated data: One desirable characteristic of multiscale representation is the decorrelation property of the wavelet coefficients of autocorrelated data at multiple scales [32], [33].

White noise processes have an autocorrelation function (ACF) with no significant spikes at early lags to indicate that the process is uncorrelated [34]. A correlated process on the other hand, such as an autoregressive process, has large ACF values at any non-zero lag. Meanwhile, wavelet coefficients that correspond to a correlated process are approximately decorrelated at multiple scales, as given in Figure 5. The first column in Figure 5 illustrates the time-domain (Figure 5a) and the detailed or wavelet signals (in Figures 5d, g, j, and m) corresponding to the following autoregressive model, $\mathrm{AR}(1)$,

$$
x_{t}=a x_{t-1}+\varepsilon,
$$

where $a=0.7$ and $\varepsilon$ is a measurement of noise with a zero mean and unit variance. Figure 5 shows the capability of wavelet decomposition to decorrelate the autocorrelated AR(1) data at multiple scales. The second column in Figure 5 illustrates that the distribution of the time domain and all detailed signals are approximately Gaussian. More information about the distribution of detailed signals will be presented later when the effect of multiscale representation on the distribution of data is discussed. The third column in Figure 5 shows that the ACF of the time-domain data (Figure 5c) has nonzero values at several lags (which indicates autocorrelation in the data as expected); the ACF of the detailed signals are approximately decorrelated (see Figures 5f, i, 1 and o).
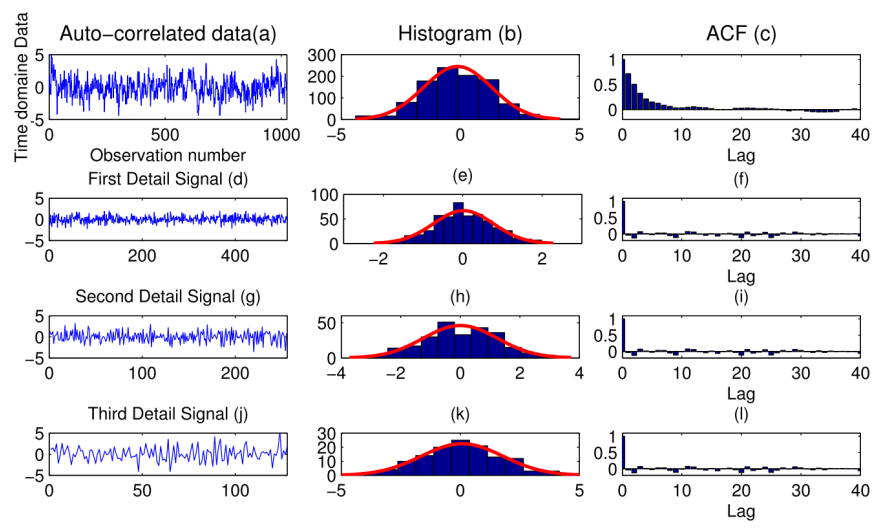

Fig. 5: Decorrelation of autocorrlated $\mathrm{AR}(1)$ data at multiple scales. 
2) Data are closer to normality at multiple scales: On important reason of the reputation of wavelet-based multiscale representation of data is its capability to transform nonGaussian data to more Gaussian on multiscale levels. For the theoretical justification on the proprieties of wavelet-based multiscale representation refer to [32], [33], [35].

Figure 6 illustrates the distributions of the detailed signals of non-Gaussian data, uniform data. It can be seen from Figure 6 that the detailed signals are close to normality (following a normal or Gaussian distribution) at multiple scales. This property of multiscale representation should help improve the efficiency of monitoring approaches when dealing with nonGaussian data.
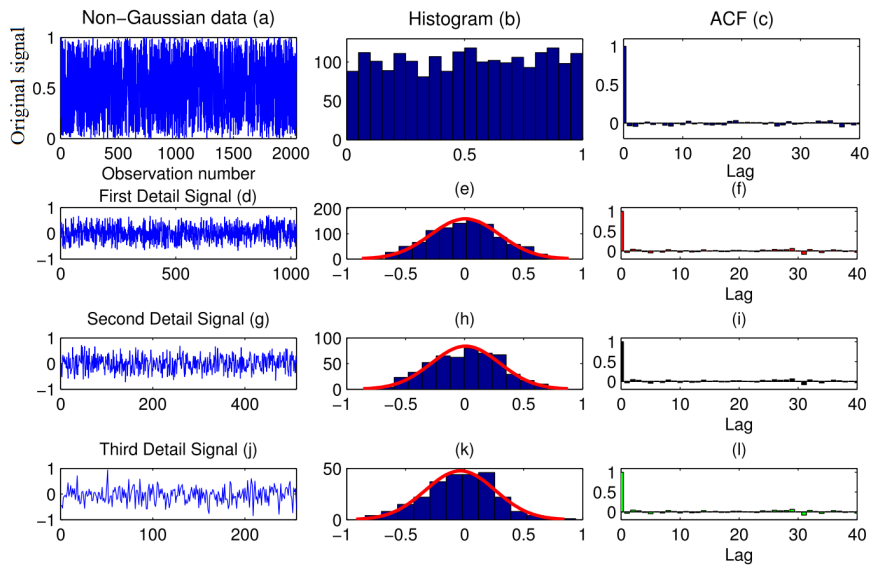

Fig. 6: Distribution and ACF of uniform data on multiple scales.

\section{Combination of PCA and WM-EWMA scheme for fault detection:}

Faults in a robot swarm usually result in the departure of the monitored swarm from its desired behavior. Real-time monitoring of a robot swarm is required for its safe and reliable operation. Based on the observed data, we would like to know whether the swarm is operating correctly or not. Practically, measurement collected from swarm robotics systems, which are usually collected through sensors, are tainted with measurements noise. The noise contaminating the collected measurements could be due to sensors degradation, disturbances, and manual errors. It should be pointed out that the presence of highly noisy measurements negatively affects the performance of a fault detection procedure. The effect of noise has to be handled such that the faults could be detected successfully. Here, we design an innovative fault detection methodology by integrating a PCA model with WM-EWMA monitoring scheme for enhanced monitoring capability of swarm robotics systems.

Overall, to monitor swarm robot, we first construct an appropriate PCA model using fault-free measurements and then compare the predicted from the model with measured (simulated) data. And (ii) fault detection is performed by evaluating the generated residuals based on the WM-EWMA monitoring scheme. In the training phase, the DWT is used to decompose the residual obtained from the PCA model into a combination of approximation and detail coefcients. Then we compute the control limits of the EWMA approach at multiple scales. At each level, detail coefficients that violate the EWMA threshold are kept in the reconstruction while the details signals below the thresholds are zeroed out. After reconstructing the signal using detailed coefficients exceed thresholds and the final approximated coefficient, we compute the EWMA threshold based on the reconstructed signal. By doing so, we keep wavelet coefficients representing relevant information for fault detection and we eliminate wavelet coefficients that do not contribute important features. In the testing stage, we utilize the thresholds obtained in the training stage to reveal any possible deviation from nominal operating conditions. This proposed monitoring scheme is diagrammatically summarized in Figure 7 and sketched in the following steps.

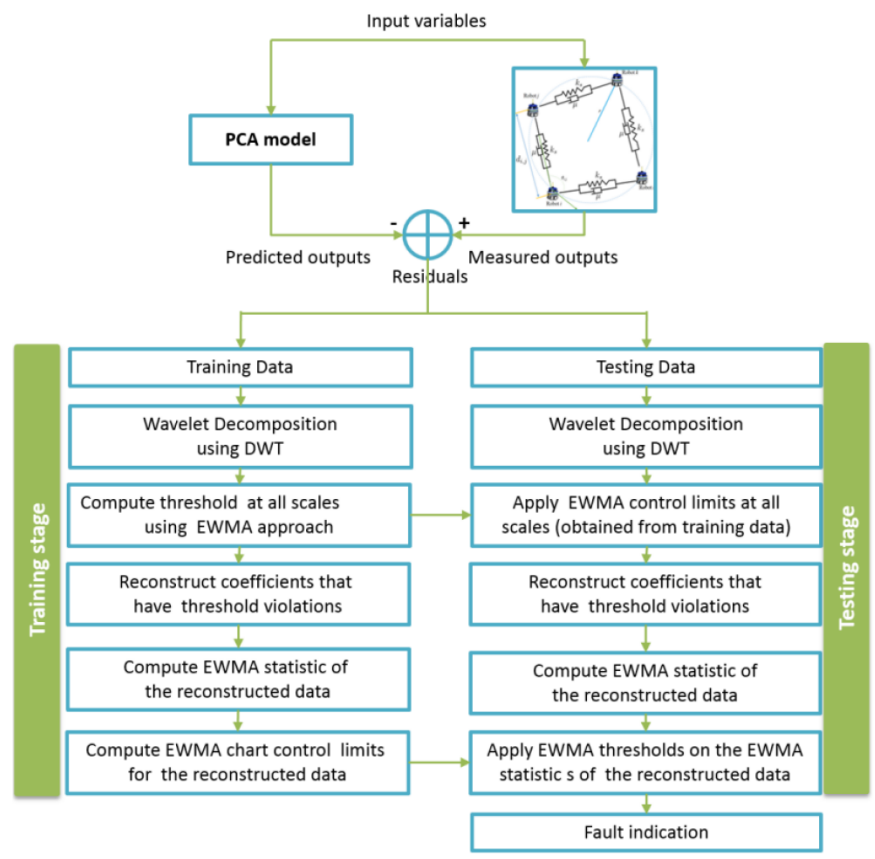

Fig. 7: A diagrammatic representation of the proposed procedure.

\section{1) Training stage:}

Step 1 Collect the training data set (fault-free data), representative of a nominal situation.

Step 2 Normalize the data to get zero mean and unit variance,

Step 3 Build PCA model based on the normalized data.

Step 4 Generate the residuals using the PCA model.

Step 5 Decompose the residuals obtained from the PCA model into wavelet coefficients using DWT. This transforms the data set from a single scale set into multiple scales set. 
Step 6 Compute the EWMA statistic and the control limits $U$ and $L$.

Step 7 Apply EWMA chart on the detail coefficients at each level and the final approximated coefficient. At each level, detail coefficients that violate the EWMA threshold are kept in the reconstruction while the details signals below the thresholds are zeroed out.

Step 8 Reconstruct data using only retained scales.

Step 9 Compute EWMA control limits for reconstructed data.

\section{2) Testing stage:}

Step 1 Normalize the testing data.

Step 2 Compute residuals using the constructed PCA model.

Step 3 Decompose the residuals into multiple scales using DWT.

Step 4 Compute the EWMA statistic and utilize the control limits obtained in the training stage for each scale.

Step 5 Reconstruct the signal using detailed coefficients that exceed EWMA thresholds and the final approximated coefficient.

Step 6 Report a fault when the WM-EWMA statistic exceeds thresholds obtained from training stage.

\section{Simulation Results}

In this study, a circle formation task is achieved using an ARGoS-based implementation of the VVC model in a swarm of 6 foot-bots. The ARGoS configuration setup of the undergoing simulations is as follows:

The foot-bots are randomly distributed in an indoor area of $10 * 6 \mathrm{~m}^{2}$ of surface. The RAB device of each foot-bot is activated within a range $D_{r}=3 \mathrm{~m}$. 5 runs of 1500 time step each are performed for the same experiment. During the experimental simulations, the data in Table.II are collected to be further used as inputs/outputs for the proposed monitoring methodology.

TABLE II: Measurements obtained from the ARGoS simulator.

\begin{tabular}{ll}
\hline Parameter & Description \\
\hline AMDE & Average mean distance error \\
GS & Group speed \\
$v_{r_{i}}$ & Right wheel forward speed \\
$v_{l_{i}}$ & Left wheel forward speed \\
$F_{i}^{v v c}$ & Virtual viscoelastic force length \\
$\angle F_{i}^{v v c}$ & Virtual viscoelastic angle \\
\hline
\end{tabular}

Two performance metrics, the group speed(GS) and the average mean distance error (AMDE) [16], [19], have been used to evaluate the efficiency of the entire swarm. Plots in Figure 8 display the evolution in time of the average of 5 runs of the two performance metrics for the 6 foot-bots. Results show that the robots started, at time step $t=500$, converging to a roughly constant AMDE and GS values. Furthermore, trajectories of the 6 foot-bots from initial positions to final positions while performing the VVC model are displayed in Figure 9. In this figure, the average of 5 runs of both the $\mathrm{X}$ and $\mathrm{Y}$ coordinate components of each foot-bot are plotted to show the final spatial distribution of the robots into a circle formation. The corresponding ARGoS-based snapshots, at different time steps $(t=0, t=250, t=500$, and $t=750)$, for the spatial distribution of the foot-bot robots are illustrated in Figure 12.

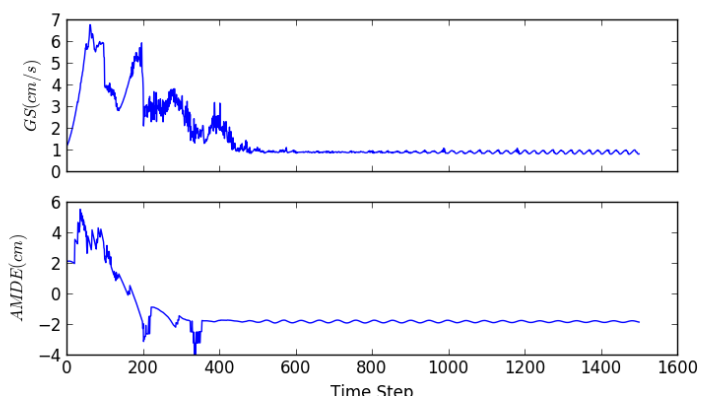

Fig. 8: The Group Speed (GS) and the Average Mean Distance Error (AMDE) performance metrics.

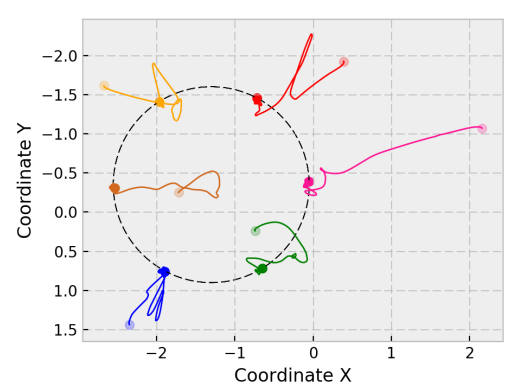

Fig. 9: Trajectories of the 6 foot-bots from intital position (Transparent color) to final position (Solid color) while performing a circle formation. Each robot is represented as a colored circle. The color of the robot is slightly transparent for the initial position when compared to the final position.

The training data collected in the absence of abnormalities would be used for building the PCA model and the developed model would be used for validating the testing data by the use of the conventional EWMA chart and its improved version WM-EWMA approach. The training data set consists of 3000 samples and 12 variables (i.e., viscoelastic force-length and viscoelastic force angle from each robot). CPV approach is employed to determine the number of PCs in the PCA model. Three PCs, which capture $96 \%$ of the useful information in the inspected swarm, are selected in order to build the PCA model. Once the model is developed from the training data, it would be used with the proposed WM-EWMA approach to detect anomalies in the testing data. Four types of anomalies in robotic swarm systems, namely bias anomaly, intermittent, 


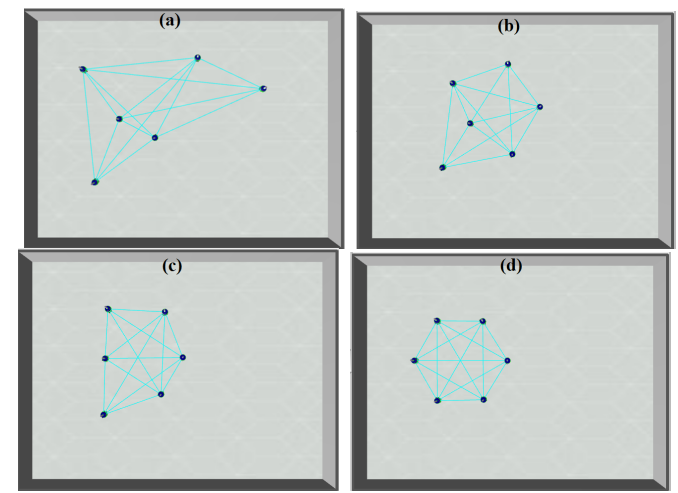

Fig. 10: ARGoS simulation at time step $\mathrm{t}=0$ (a), $\mathrm{t}=250$ (b), $\mathrm{t}=500$ (c) and $\mathrm{t}=750$ (d).

random walk, and complete stop faults would be introduced into the testing data.

\section{A. Detection results}

Now, we describe how we generate faulty data in the encoder/sensor sub-components of one or more robots. A measurement, $X_{m}(t)$, is commonly modeled as follow:

$$
X_{m}(t)=X_{r}(t)+F(t),
$$

where $X_{r}(t)$ is the true measurement value and $F(t)$ is a measure of a systematic error at time $t$. The system error $F(t)$ is mainly caused by a malfunction, corresponding to different fault mode. To this end, we use the following faults modes in the motor and the RAB sensors:

Abrupt fault mode:: Bias faults are kinds of faults commonly existing in practice. Generally, in this mode the correct measurement has a constant deviation, i.e.

$$
X_{m}(t)=\left\{\begin{array}{ll}
X_{r}(t) & \text { if } t<t_{\mathrm{f}} \\
X_{r}(t)+c & \text { if } t \geqslant t_{\mathrm{f}}
\end{array},\right.
$$

where $t_{f}$ is the time of the occurrence of the fault, and $c$ is a bias constant

Stop fault mode:: A stop failure mode refers to the complete dysfunctioning of a sub-component. This model can be mainly modeled as follow:

$$
X_{m}(t)= \begin{cases}X_{r}(t) & \text { if } t<t_{\mathrm{f}} \\ 0 & \text { if } t \geqslant t_{\mathrm{f}}\end{cases}
$$

Random walk faults: A Random walk fault refers to a fault where the inspected robot moves randomly. This fault is simulated by contaminating the measurements of the viscoelastic force-length of the robot by a random Gaussian noise with a variance $\sigma^{2}$.

The faults types considered in this study have been injected to the true measures of the motor and the $\mathrm{RAB}$ sensor of one or more foot-bots while a swarm of six foot-bots is performing

$$
X_{m}(t)= \begin{cases}X_{r}(t) & \text { if } t<t_{\mathrm{f}} \\ X_{r}(t)+N\left(0, \sigma^{2}\right) & \text { if } t \geqslant t_{\mathrm{f}}\end{cases}
$$

a circle formation task. All partially faulty data were injected in real time during an ARGoS based simulation of the desired task. The performance of detection procedures is quantitatively compared using the following metrics: the missed detection rate (MDR) and the false alarm rate (FAR). The smaller the FAR and MDR are, the better detection is achieved.

1) Cases with abrupt faults: In this subsection, the effectiveness of the developed approach in detecting abrupt change faults in a robot swarm is illustrated. We introduced a bias to the measurements of the viscoelastic force-length of the first robot from sample times 150 to 200. The amplitude of the introduced bias is equal to $10 \%$ of the total variation found in the raw data. Recall that the virtual viscoelastic force is essentially associated with the RAB device and that by adding a deviation to such a force, either the neighbors of the robot are misperceived, or the data (velocities) sent by neighbors are misperceived. The performances of conventional EWMA chart and its multiscale extension, WM-EWMA, in detecting a fault in swarm robots are studied under noisy environment. To do so, let us consider scenarios when the measurement sensors were corrupted by noise. We modeled measurements noise with Gaussian distribution with zero mean and a standard deviation, $\sigma$, but this time we investigate different values of $\sigma=0.3,0.9,1.7$. The results of the two charts for testing data with different noise levels, $\sigma=0.3$ ( low noisy data), $\sigma=0.9$ (moderate noisy data), and $\sigma=1.7$ (high noisy data) are demonstrated in Figures 14, 13 and 12, respectively.

From this three examples, we can note that the introduced noise levels have an undesirable impact in the detection performance of the conventional PCA-EWMA approach. In the case of large noise level, it is unfeasible to use EWMA approach for fault detection. The simulated fault has been masked by noise measurement, hence the conventional EWMA becomes incapable to detect this abrupt fault and result in several false alarms and missed detection (see Figure 14). Fortunately, the wavelet-based multiscale strategy, which is a powerful data analysis tool since it can extract useful data simultaneously from time and frequency domains, is available for unmasking the hidden features (faults) lost in noisy data. The PCA-based WM-EWMA approach is relatively robust to these noise levels contaminating the sensors measurements, so it can be utilized to handle noisy data. Figure 14(b) indicates that the false alarms are significantly reduced when applying WM-EWMA chart.

These examples in Figures (13-14) illustrate how a large amount of noise masked the important feature and faults in data. To make statistically valid conclusions on the robustness of these two monitoring charts against noise measurements, a Monte Carlo simulation with 1000 realizations is accomplished in each scenario (Table III). From the table, it can be seen that the highly noisy data have a dramatic impact on the detection performance of the conventional EWMA chart, and the impact seems less serious in cases with low noisy data. 

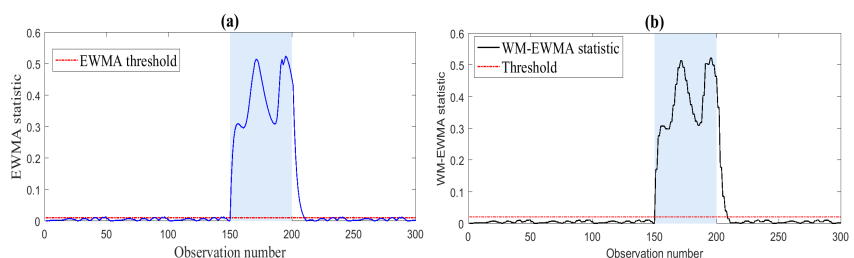

Fig. 11: (a) EWMA and (b) WM-EWMA schemes in the case of an abrupt fault, noiseless data.
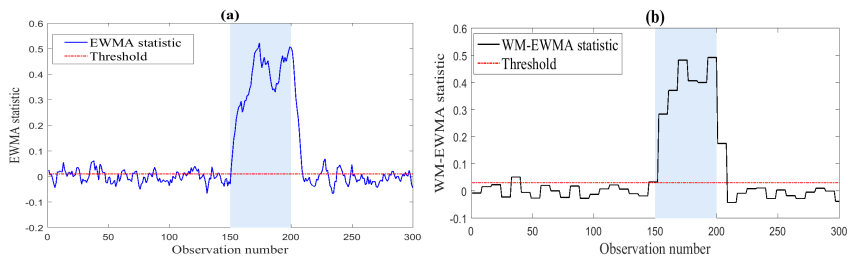

Fig. 12: (a) EWMA and (b) WM-EWMA schemes in the case of an abrupt fault, low noisy data example.

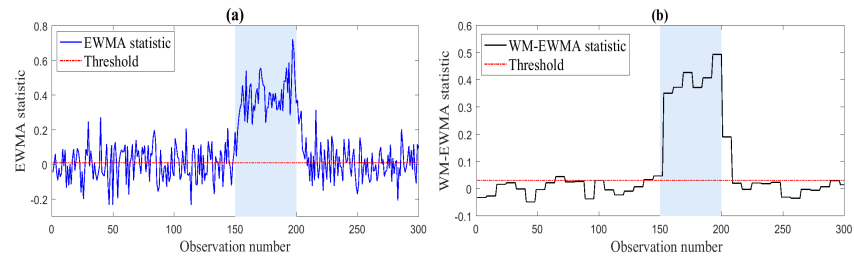

Fig. 13: (a) EWMA and (b) WM-EWMA schemes in the case of an abrupt fault, moderate noisy data example.
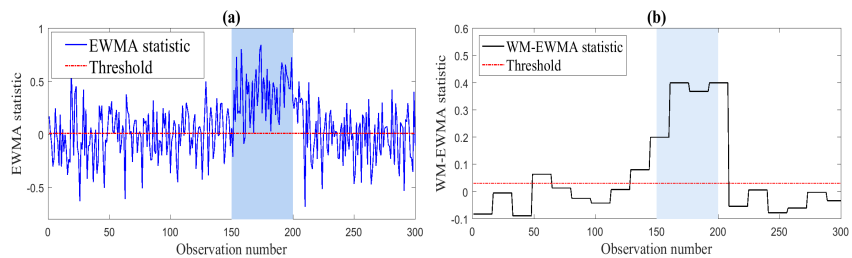

Fig. 14: (a) EWMA and (b) WM-EWMA schemes in the case of an abrupt fault, high noisy data example.

Also, Table III confirms the robustness to noise measurements and sensitivity to abrupt faults of the proposed PCA-based WM-EWMA approach.

TABLE III: FAR and MDR values of the EWMA and MWEWMA schemes in various scenarios of noisy data.

\begin{tabular}{|c|c|c||c|c|}
\hline \multicolumn{3}{|c}{ EWMA chart } & \multicolumn{2}{c|}{ WM-EWMA chart } \\
\hline$\sigma$ & FAR & MDR & FAR & MDR \\
\hline 0.3 & 7.33 & 1.49 & 0.33 & 0.80 \\
\hline 0.9 & 44.20 & 2.25 & 6.96 & 1.95 \\
\hline 1.7 & 74.70 & 9.45 & 9.44 & 2.47 \\
\hline
\end{tabular}

2) Cases with intermittent faults: In this case, the robustness to sensor noise and sensitivity to intermittent faults of the proposed approach have been investigated. To do so, an intermittent fault has been introduced into the raw data, with a bias of amplitude $40 \%$ of the total variation of raw measurements from time instant 50 to 100 and bias of amplitude $10 \%$ for time instant 150 to 200 . This again suggests a repeated presence of a complete sensor offset in the RAB device (a RAB sensor fault); which could due to erroneous data communicated by neighbors or to noisy measurements (range and bearing). To illustrate the robustness of the proposed procedure to noise measurements, in this scenario we add random noises with different levels following the normal distributions to measured data. Figures (15-17) illustrate the monitoring results of EWMA and WM-EWMA approaches for the low, moderate and high noise levels cases in which the raw data are added with three levels of noise low $(\sigma=0.3)$, moderate noisy data $(\sigma=0.9)$ and highly noisy data $(\sigma=1.7)$, respectively. It can be seen that in the high amount of noise the conventional PCA-EWMA becomes insensitive to faults because it is not sufficiently robust to noise measurements(Figures 16(a)-17(a)). The fault signal is lost in highly noisy data when using EWMA approach, and it delivers poor and misleading results. On the other hand, the proposed PCA-based WM-EWMA provides more efficient and robust detection results compared to the conventional EWMA approach.
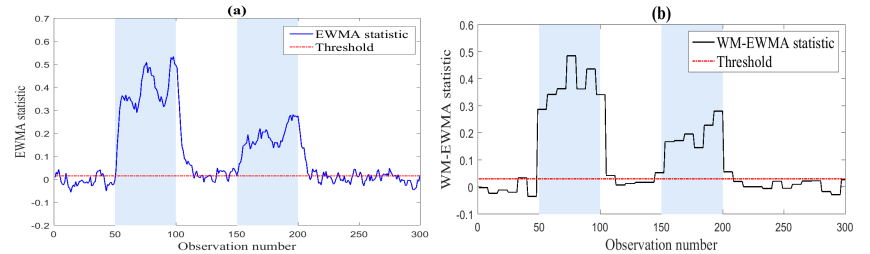

Fig. 15: (a) EWMA and (b) WM-EWMA schemes in the case of an intermittent fault, low noisy data example.
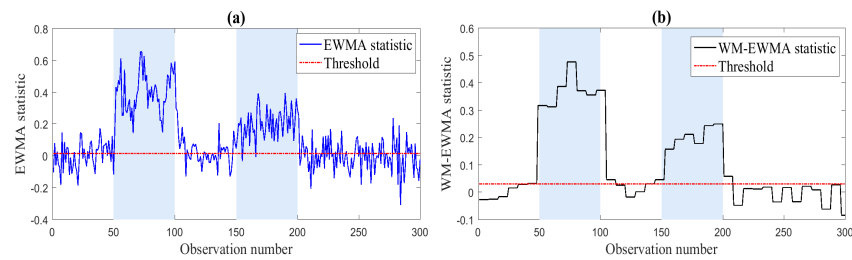

Fig. 16: (a) EWMA and (b) WM-EWMA schemes in the case of an intermittent fault, moderate noisy data example.

Table IV summarize the performance of the two approaches in term of the average of FAR and MDR computed using a Monte Carlo simulation with 1000 replicated simulations. Results in Table IV confirms the superiority of the WMEWMA approach compared to conventional EWMA approach shows that the proposed approach results in lowest FAR and MDR compared to the conventional one. Based on this result, the proposed WM-EWMA chart is more robust to noise as compared to the conventional EWMA. 

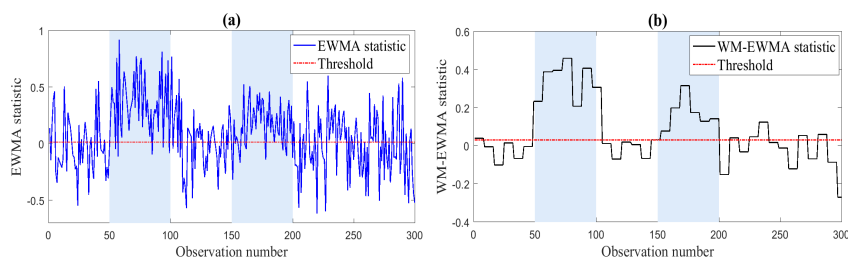

Fig. 17: (a) EWMA and (b) WM-EWMA schemes in the case of an intermittent fault, high noisy data example.

TABLE IV: FAR and MDR values of the EWMA and MWEWMA schemes in various scenarios of noisy data.

\begin{tabular}{|c|c|c||c|c|}
\hline \multicolumn{3}{|c}{ EWMA chart } & \multicolumn{2}{c|}{ WM-EWMA chart } \\
\hline$\sigma$ & FAR & MDR & FAR & MDR \\
\hline 0.3 & 10.70 & 2.40 & 1.20 & 0.90 \\
\hline 0.9 & 49.10 & 4.38 & 8.96 & 3.20 \\
\hline 1.7 & 78.15 & 14.91 & 12.50 & 7.11 \\
\hline
\end{tabular}

3) Cases with random walk faults: This case focuses on the capability of the PCA-based WM-EMWA strategy in detecting random walk faults in a robot swarm. Here, we consider that the first robot moves randomly and do not follow the other robots. This fault can be generated due to noises in the RAB device of the robot. To simulate a random walk fault, we injected random Gaussian noise with a standard deviation of $\sigma=0.5$ and zero mean in measurements of the viscoelastic force-length of the first robot from time instant 200. The detection analysis has been performed for the data with different noise levels (see Figures (18-20)). For the moderate and relatively high noisy data, the performances of the conventional PCA-EWMA and proposed PCA-based WM-EWMA techniques can be seen in Figures 19 and 20, respectively. It could be noted that the WM-EWMA chart provides a clear improvement over conventional EWMA chart in term of decreasing the FAR and MDR. The detection results clearly indicate the superiority and the robustness of proposed strategy in handling random walk faults.
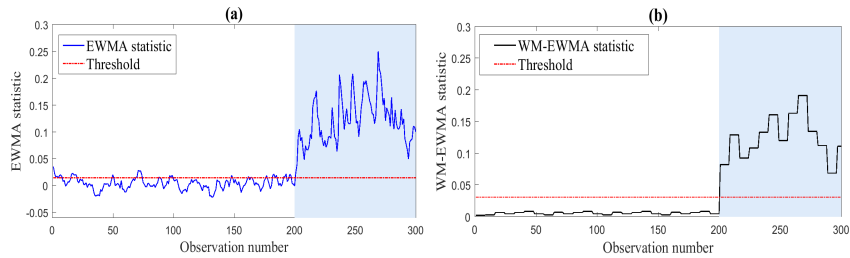

Fig. 18: (a) EWMA and (b) WM-EWMA schemes in the case of a random walk fault, low noisy data example.

To make statistically reliable conclusions on the robustness of these two monitoring charts against noise measurements, a Monte Carlo simulation with 1000 realizations is accomplished in each scenario, and the results in term of FAR and MDR are summarized in Table $\mathrm{V}$. These results prove that integrating the EWMA chart with the wavelet-based multiscale representation

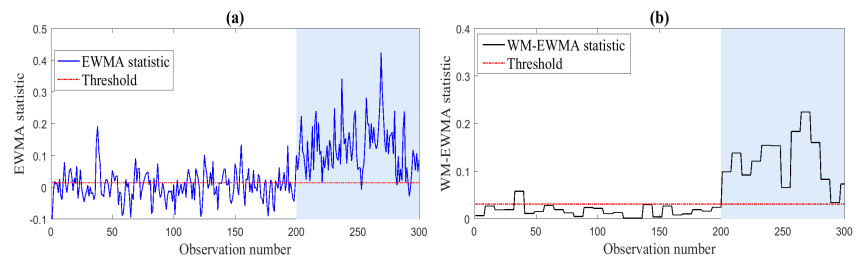

Fig. 19: (a) EWMA and (b) WM-EWMA schemes in the case of a random walk fault, moderate noisy data example.
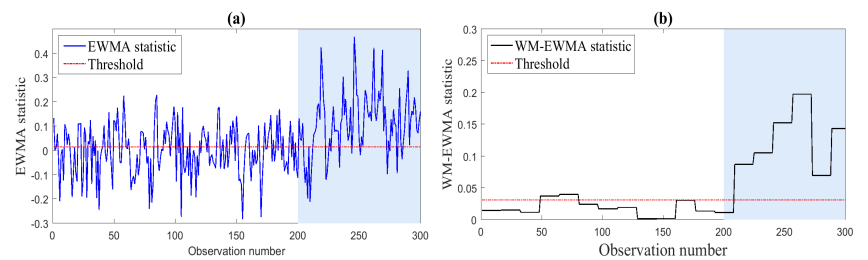

Fig. 20: (a) EWMA and (b) WM-EWMA schemes in the case of a random walk fault, high noisy data example.

approach enhances the quality of fault detection by reducing false alarms (see Table V).

TABLE V: FAR and MDR values of the EWMA and MWEWMA schemes in various scenarios of noisy data.

\begin{tabular}{c|c|c|c|c|}
\multicolumn{3}{|c|}{ EWMA chart } & \multicolumn{2}{c}{ WM-EWMA chart } \\
$\sigma$ & FAR & MDR & FAR & MDR \\
\hline 0.3 & 7.23 & 3.49 & 0.12 & 0.20 \\
\hline 0.9 & 37 & 7.50 & 6.40 & 1.10 \\
\hline 1.7 & 76.07 & 17.91 & 8.10 & 1.80 \\
\hline
\end{tabular}

4) Cases with complete stop faults: Here, a complete stop fault in a robot swarm is considered to show the effectiveness of the proposed methodology. This suggests a complete failure in both the motor actuator and the RAB device of the faulty robot, preventing it from moving, sending or receiving data. In this scenario, we observe a complete stop fault, i.e., the viscoelastic force of the first robot is zeroed from the 200 of the time instant. Next, we investigate the impact of the noisy data on the detection capacity of the two approaches in the presence of temporary shading faults. Figures (21-23) illustrate respectively the results of the two charts for testing data with different noise levels low noisy, moderate noisy and high noisy datasets. From the results of the conventional EWMA approach, it can be seen that introduced faults are undetectable because faults are buried in noise (i.e., high amount of noise in the data masked important features and faults). Due to the large level noise, it is not easy to accurately detect the introduced faults with the EWMA chart. As seen in the results, the proposed approach has a better performance and robustness to measurements noise compared to the conventional EWMA test in detecting the presence of complete stop fault in a robot swarm.

Table VI shows the detection performance (i.e., FAR and 


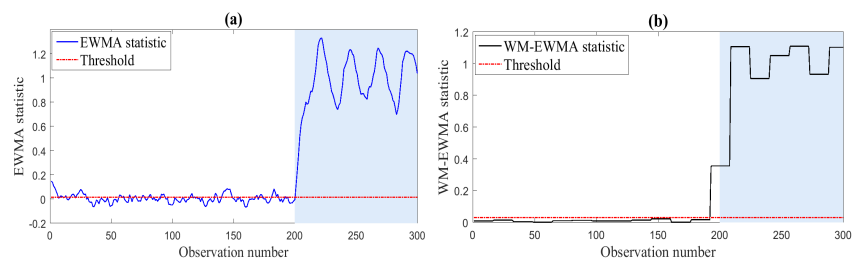

Fig. 21: (a) EWMA and (b) WM-EWMA schemes in the case of a complete stop fault, low noisy data example.

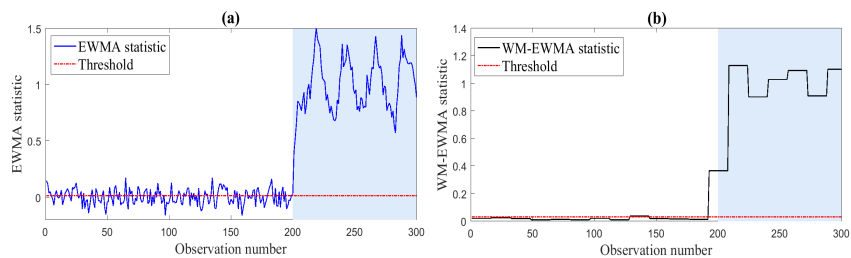

Fig. 22: (a) EWMA and (b) WM-EWMA schemes in the case of a complete stop fault, moderate noisy data example.
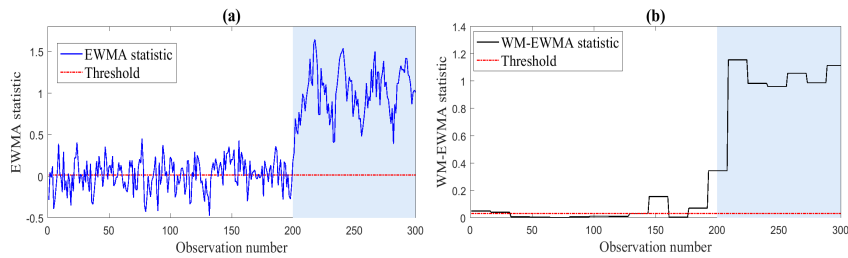

Fig. 23: (a) EWMA and (b) WM-EWMA schemes in the case of a complete stop fault, high noisy data example.

MDR) of the proposed approach and the conventional PCAEWMA approach. Results in Table VI clearly indicating the WM-EWMA strategy is more accurate in detecting a complete stop fault. The proposed approach can detect complete stop faults which are missed by the conventional approach and with much fewer false alarms. In other word, the proposed approach is more robust to noise measurements compared to the conventional PCA-EWMA chart.

TABLE VI: FAR and MDR values of the EWMA and MWEWMA schemes in various scenarios of noisy data.

\begin{tabular}{c|c|c||c|c|}
\hline \multicolumn{3}{|c}{ EWMA chart } & \multicolumn{2}{c|}{ WM-EWMA chart } \\
\hline$\sigma$ & FAR & MDR & FAR & MDR \\
\hline 0.3 & 6.40 & 0.20 & 0.40 & 0.20 \\
\hline 0.9 & 39.28 & 1.24 & 6.75 & 0.37 \\
\hline 1.7 & 57.30 & 1.70 & 8.50 & 0.94 \\
\hline
\end{tabular}

\section{CONCLUSION}

Despite the flexibility and the scalability of swarm robotics systems, undesired results can occur while performing the planned task using such robotic systems. Failures are inevitable when dealing with robot swarms due to external interferences, unexpected collisions between robots, component faults, software bugs and even broken communication links. Hence, the coordinated activity of robot swarms needs to be monitored to keep robot swarms operating at peak performance. Data from sensors in the robot swarm are generally contaminated with noise, which masks important features in the data and deteriorates the fault detection capability of a monitoring system. In this paper, the robust fault detection problem in a robot swarm under noisy environment is studied. The proposed approach to fault detection in robotic swarm systems combines the advantages of univariate PCA, EWMA chart and wavelet-based multiresolution denoising. PCA model is used to generate residuals for fault detection. The new detection scheme, WMEWMA, merges the benefits of multiscale representation of data and those of the EWMA scheme to suitably detect faults. Here, multiscale representation of data using wavelets, which is able to decouple features/noise, has been used to enhance fault detection performance minimizing uncertainties and noise effects. Superiority and robustness of the proposed approach to noise measurements compared to that of the conventional PCA-EWMA have been tested by simulated data obtained from the ARGoS simulator. The approach showed good robustness abilities to noise measurements in terms of false alarms while maintaining good fault detection performances.

To further fault detection in a robot swarm, in future works, we plan to develop an automated WM-EWMA approach by determining the optimal number of decomposition in DWT automatically that provides suitable fault detection performances. Also, we plan to test and validate the developed approach using experimental data.

\section{ACKNOWLEDGMENT}

The work presented in this publication was supported by the King Abdullah University of Science and Technology (KAUST) Office of Sponsored Research (OSR) under Award No: OSR-2015-CRG4-2582.

\section{REFERENCES}

[1] B. Khaldi, F. Harrou, F. Cherif, and Y. Sun, "Self-organization in aggregating robot swarms: A DW-KNN topological approach," Biosystems, vol. 165, pp. 106-121, 2018.

[2] M. Dorigo, M. Birattari et al., "Swarm intelligence." Scholarpedia, vol. 2, no. 9, p. 1462, 2007.

[3] B. Khaldi and F. Cherif, "An overview of swarm robotics: Swarm intelligence applied to multi-robotics," International Journal of Computer Applications, vol. 126, no. 2, 2015.

[4] J. Timmis, A. R. Ismail, J. D. Bjerknes, and A. F. Winfield, "An immune-inspired swarm aggregation algorithm for self-healing swarm robotic systems," Biosystems, vol. 146, pp. 60-76, 2016.

[5] J. D. Bjerknes and A. F. Winfield, "On fault tolerance and scalability of swarm robotic systems," in Distributed autonomous robotic systems. Springer, 2013, pp. 431-444.

[6] A. Millard, J. Timmis, and A. Winfield, "Run-time detection of faults in autonomous mobile robots based on the comparison of simulated and real robot behaviour," in 2014 IEEE/RSJ International Conference on Intelligent Robots and Systems. IEEE, 2014, pp. 3720-3725.

[7] A. Christensen, R. OGrady, and M. Dorigo, "From fireflies to faulttolerant swarms of robots," IEEE Transactions on Evolutionary Computation, vol. 13, no. 4, pp. 754-766, 2009. 
[8] E. Skoundrianos and S. Tzafestas, "Finding fault-fault diagnosis on the wheels of a mobile robot using local model neural networks," IEEE Robotics \& Automation Magazine, vol. 11, no. 3, pp. 83-90, 2004.

[9] A. Christensen, R. OǴrady, M. Birattari, and M. Dorigo, "Fault detection in autonomous robots based on fault injection and learning," Autonomous Robots, vol. 24, no. 1, pp. 49-67, 2008.

[10] M. Mokhtar, R. Bi, J. Timmis, and A. Tyrrell, "A modified dendritic cell algorithm for on-line error detection in robotic systems," in IEEE Congress on Evolutionary Computation. IEEE, 2009, pp. 2055-2062.

[11] D. Tarapore, P. Lima, J. Carneiro, and A. Christensen, "To err is robotic, to tolerate immunological: fault detection in multirobot systems," Bioinspiration \& biomimetics, vol. 10, no. 1, p. 016014, 2015.

[12] B. Jakimovski and E. Maehle, "Artificial immune system based robot anomaly detection engine for fault tolerant robots," in International Conference on Autonomic and Trusted Computing. Springer, 2008, pp. 177-190.

[13] F. Harrou, L. Fillatre, and I. Nikiforov, "Anomaly detection/detectability for a linear model with a bounded nuisance parameter," Annual Reviews in Control, vol. 38, no. 1, pp. 32-44, 2014.

[14] S. Yin, S. X. Ding, X. Xie, and H. Luo, "A review on basic data-driven approaches for industrial process monitoring," IEEE Transactions on Industrial Electronics, vol. 61, no. 11, pp. 6418-6428, 2014.

[15] B. Khaldi, F. Harrou, F. Cherif, and Y. Sun, "Monitoring a robot swarm using a data-driven fault detection approach," Robotics and Autonomous Systems, vol. 97, pp. 193-203, 2017.

[16] B. Khaldi and F. Cherif, "Swarm robots circle formation via a virtual viscoelastic control model," in 8th International Conference on Modelling, Identification and Control (ICMIC-2016). IEEE, 2016.

[17] H. Choi and J. Jeong, "Despeckling images using a preprocessing filter and discrete wavelet transform-based noise reduction techniques," IEEE Sensors Journal, vol. 18, no. 8, pp. 3131-3139, 2018.

[18] B. Khaldi and F. Cherif, "Swarm robots circle formation via a virtual viscoelastic control model," in $8 t$ h International Conference on Modelling, Identification and Control (ICMIC-2016). IEEE, 2016.

[19] B. Khaldi, and F. Cherif, "A virtual viscoelastic based aggregation model for self-organization of swarm robots system," in Conference Towards Autonomous Robotic Systems. Springer, 2016, pp. 202-213.

[20] G. Pini, M. DORIGO, and M. BIRATTARI, "Towards collective robotics in a 3d space: simulation with hand-bot robots," 2009.

[21] E. Ferrante, A. Turgut, C. Huepe, A. Stranieri, C. Pinciroli, and M. Dorigo, "Self-organized flocking with a mobile robot swarm: a novel motion control method," Adaptive Behavior, p. 1059712312462248, 2012.

[22] C. Pinciroli, V. Trianni, R. OGrady, G. Pini, A. Brutschy, M. Brambilla, N. Mathews, E. Ferrante, G. D. Caro, and F. Ducatelle, "ARGoS: a modular, parallel, multi-engine simulator for multi-robot systems," Swarm intelligence, vol. 6, no. 4, pp. 271-295, 2012.

[23] M. R. Mowla, S.-C. Ng, M. S. Zilany, and R. Paramesran, "Singletrial evoked potential estimation using iterative principal component analysis," IEEE Sensors Journal, vol. 16, no. 18, pp. 6955-6960, 2016.

[24] P. B. Garcia-Allende, O. M. Conde, J. Mirapeix, A. M. Cubillas, and J. M. López-Higuera, "Data processing method applying principal component analysis and spectral angle mapper for imaging spectroscopic sensors," IEEE Sensors Journal, vol. 8, no. 7, pp. 1310-1316, 2008.

[25] A. Perera, N. Papamichail, N. Bârsan, U. Weimar, and S. Marco, "On-line novelty detection by recursive dynamic principal component analysis and gas sensor arrays under drift conditions," IEEE Sensors Journal, vol. 6, no. 3, pp. 770-783, 2006.

[26] D. C. Montgomery, "Introduction to statistical quality control," John Wiley\& Sons, New York, 2005.

[27] F. Harrou, Y. Sun, M. Madakyaru, and B. Bouyedou, "An improved multivariate chart using partial least squares with continuous ranked probability score," IEEE Sensors Journal, vol. 18, no. 16, pp. 67156726, 2018.
[28] F. Harrou and M. Nounou, "Monitoring linear antenna arrays using an exponentially weighted moving average-based fault detection scheme," Systems Science \& Control Engineering: An Open Access Journal, vol. 2, no. 1, pp. 433-443, 2014.

[29] R. Ganesan, T. Das, and V. Venkataraman, "Wavelet-based multiscale statistical process monitoring: A literature review," IIE transactions, vol. 36, no. 9, pp. 787-806, 2004.

[30] R. Gao and R. Yan, Wavelets: Theory and applications for manufacturing. Springer Science \& Business Media, 2010.

[31] G. Strang, "Wavelets and dilation equations: A brief introduction," SIAM review, vol. 31, no. 4, pp. 614-627, 1989.

[32] H. Aradhye, B. Bakshi, and a. J. D. R.A. Strauss, "Multiscale SPC using wavelets: theoretical analysis and properties," AIChE Journal, vol. 49, no. 4, pp. 939-958, 2003.

[33] X. Chang and M. L. Stein, "Decorrelation property of discrete wavelet transform under fixed-domain asymptotics," IEEE Transactions on Information Theory, vol. 59, no. 12, pp. 8001-8013, 2013.

[34] G. Box, G. Jenkins, G. Reinsel, and G. Ljung, Time series analysis: forecasting and control. John Wiley \& Sons, 2015.

[35] D. L. Donoho and J. M. Johnstone, "Ideal spatial adaptation by wavelet shrinkage," biometrika, vol. 81, no. 3, pp. 425-455, 1994. 